\title{
IL MITO DEL PAESE DI CUCCAGNA E LA PUBBLICITÀ TELEVISIVA ITALIANA
}

\begin{abstract}
A bstract. Kornacka Barbara, Il mito del paese di Cuccagna e la pubblicità televisiva italiana [The myth of the land of Cuccagna and Italian television advertisement]. Studia Romanica Posnaniensia, Adam Mickiewicz University Press, Poznań, vol. XXXIII : 2006, pp. 17-30. ISBN 83-232-1643-6, ISSN 0J37-2475.
\end{abstract}

The article compares medieval and Renaissance literary descriptions of the mythic land of eternal bliss and prosperity Cuccagna with pictures and texts of Italian television advertisement of the nineties. The characteristic moments of these descriptions such as, for example, abundance, satisfying sensual needs, celebrating and feasting as well as the evaluation of the described phenomena, in these distant texts of culture, are very similar to each other.

Italian television advertisement appears as another contemporary image of the land of bliss Cuccagna.

\section{INTRODUZIONE}

Il mito del paese di Cuccagna, da secoli e tuttora presente nella cultura italiana e nella mentalità degli italiani, è un elogio della carnalità intesa come messa in rilievo del corpo e dei suoi bisogni nonché dell'appagamento dei desideri corporali. Crediamo che ci siano diversi fattori che accomunano questa tradizionale e popolaresca visione del paradiso terrestre del piacere corporale e le immagini della pubblicità televisiva la quale, pur moderna e, dal punto di vista tecnologico, più adeguata al linguaggio dei nostri tempi, trasmette alle masse uguali messaggi e risponde a uguali nostalgie.

La Cuccagna, scrive P. Burke „è una visione che assimila la vita a un lungo Camevale, mentre il Carnevale, a sua volta, è una Cuccagna transitoria, ma con la stessa esaltazione del cibo e dei rovesciamenti"". Secondo P. Camporesi invece il carnevale e il paese di Cuccagna ,vengono a confondersi nel gigantesco mito della povera gente secondo il quale soltanto dai morti, dagli spiriti e dal diavolo è possibile ottenere felicità e ricchezza, non dai potenti e dai vivi"'. Il paese di Cuccagna si configura come un paese leggendario, come un paese lontano, felice, sognato,

${ }^{1}$ P. Burke, Cultura popolare nell'Europa moderna. Mondadori, Milano 1980, pp. 185-186.

${ }^{2}$ P. Camporesi, Il paese della fame, Il Mulino, Bologna 1978, p. 206. 
dove l'uomo e, soprattutto, quello misero ed affamato, poteva trovare tutti i beni della terra. „Il viaggio verso Cuccagna, verso una terra che non conosce angustie, carestie, dolori, malattie, oltraggi; il pellegrinaggio verso l'Eden mitico alla ricerca della smarrita età dell'oro, (...) corrisponde in qualche modo al viaggio che le droghe rituali hanno sempre offerto alle misere genti d'altri paesi e d'altri continenti schiacciate dalla durezza della vita e dalle fatiche dell'esistenza" - scrive in proposito $\mathrm{P}$. Camporesi ${ }^{3}$. Una delle più antiche descrizioni del paese di Cuccagna racconta che „le case vi sono fatte di pesci, di salsicce e d'altre cose ghiotte. Le oche grasse si vanno avvolgendo per le vie, arrostendosi da se stesse, accompagnate dalla bianca agliata, e vi son tavole sempre imbandite d'ogni vivanda, a cui ognuno può assidersi liberamente, e mangiare di ciò che meglio gli aggrada, senza mai pagare un quattrino di scotto. Da bere porge un fiume, il quale è mezzo di vino rosso, e mezzo di vino bianco. In quella terra il mese è di sei settimane, e vi si celebrano quattro pasque, e quadruplicate sono l'altre feste principali, mentre la quaresima viene solo una volta ogni vent'anni. I denari si trovano, come i sassi, per terra; ma non bisognano, perché nessuno compra o vende, e tutto quanto è necessario alla vita si dà per nulla. Le dinne che vi sono altro non chiedono che fare altrui piacere, e ci è la fontana di gioventừ"

In seguito metteremo in luce alcune fonti letterarie del mito del paese di Cuccagna e poi confronteremo le caratteristiche di questa terra felice e leggendaria dove si realizzano tutti i desideri del corpo con le immagini della pubblicità televisiva italiana degli ultimi dieci anni, la quale ci sembra una realizzazione contemporanea, adeguata all'uomo contemporaneo, del mito e dei modi di immaginarlo.

Le pubblicità in seguito analizzate sono state prodotte e messe in onda nel corso degli anni novanta del secolo scorso e appartengono al repertorio di spot delle grandi marche italiane, più conosciute al mondo e spettacolari. D'altra parte abbiamo ritenute significative le pubblicità dei prodotti „,nostrani”, locali che funzionano solamente sul mercato italiano e quindi i cui filmati pubblicitari sono destinati al ristretto pubblico italiano e solo da esso pienamente percepito.

\section{LE FONTI LETTERARIE DEL MITO DEL PAESE DI CUCCAGNA}

Nella letteratura popolare vive, muore e rinasce tutta una serie di temi, o meglio, di schemi narrativi che, risolvendosi molte volte più o meno compiutamente, nella poesia, nella fiaba, nella leggenda o nel canto fioriscono presso le civiltà e i popoli più diversi ${ }^{5}$. Tra questi motivi vi è il paese di Cuccagna ${ }^{6}$.

\footnotetext{
${ }^{3}$ Ibidem.

${ }^{4}$ Barbazan-Méon, Fabliaus et contes, vol. IV, pp. 175-181; riassunto da A. Graf, Il paese di Cuccagna e i paradisi artificiali, in: Miti. leggende e superstizioni del Medio Evo, Loescher, Torino 1892, I, pp. 232-233.

${ }^{5} \mathrm{G}$. Cocchi ara, Il mondo alla rovescia, Boringhieri, Torino 1981, p. 3.

${ }^{6}$ Cfr. ibidem, p. 159. Sul paese di Cuccagna cfr. anche A. Zenatti, l'introduzione alla Storia di Campriano contadino, Romagnoli, Bologna 1884, pp. LVIII-LXII; F. Novati, Il paese che non si
} 
L'etimologia del nome di Cuccagna ${ }^{7}$ non è molto sicura. Graf ricorda che un abbas Cucaniensis è già in una poesia goliardica composta probabilmente fra il 1162 e il 1164 e che Cuccagna fu il nome di un castello ancora in parte esistente presso Treviso. Inoltre, tal nome ricorre già in documenti del $1142 \mathrm{e}$ un Warnerius de Cuccagna compariva in una carta del $1188^{8}$.

In Italia una delle più antiche testimonianze sul paese di Cuccagna ci è data da Boccaccio nel suo Decameron (VIII, 3), dove Cuccagna prende il nome di Bengodi, le meraviglie del quale Maso racconta a Calandrino. Inoltre, un sonetto attribuito a Burchiello finisce con questi versi:

Allo passando alzò la sua visiera

E donò lor mille buoni trovi

Domandando le chiavi

Di Mongibal, di Roma e di Romagna

Per fare armata contro la Cuccagna

Una descrizione più complessa risale agli ultimi anni del Quattrocento ed è intitolata Historia nuova della Città di Cuccagna, data in luce da Alessandro da Siena e Bartolomeo suo compagno. Un accenno al paese di Cuccagna lo troviamo in una lettera di Andrea Calmo del 1547-1566. Sempre nel Cinquecento Tommaso Garzoni, nell'opera Piazza universale di tutte le professioni e mestieri nella rubrica che egli intitola De' pellegrini o viandanti, ci descrive il paese di Cuccagna come una delle fanfaluche che al ritorno dei loro fantastici viaggi raccontano i pellegrini. Nel Cinquecento si nota comunque un particolare fiorire del mito del paese di Cuccagna, oggetto di molte narrazioni del tempo. La sua popolarità in gran parte fu dovuta alla Storia di Campriano contadino inclusa in Capricciosi ragionamenti $\mathrm{di}$ Pietro Aretino. Una descrizione del paese di Cuccagna ci è tramandata da un ebreo in un poemetto del sedicesimo secolo intitolato Lo aviso et vision venuto alli barberi. Faustino Terdocio dello stesso Cinquecento in un suo satirico Testamento vuole che la sua anima voli come farfalla nel Paradiso che ha tutte le caratteristiche

trova, La domenica letteraria, IV, n. 2, (15 marzo 1885); V. Rossi, Il paese di Cuccagna nella letteratura italiana, in: Le lettere di messer Andrea Calmo, Torino 1888, Appendice II, pp. 398-410; A. Graf, Il paese di Cuccagna e i paradisi artificiali, in: Miti, leggende e superstizioni del Medio Evo. op. cit., pp. 229-238; G. Cocchiara, Il paese di cuccagna. L'evasione dalla realtà nella fantasia popolare, in: Il paese di Cuccagna e altri studi di folclore, Einaudi, Torino 1956, pp. 159-187; $\mathrm{G}$. Costa, La leggenda dei secoli d'oro nella letteratura italiana, Laterza, Bari 1972; E.M. Ackermann, Das Schlaraffenland in German Literature, and Folksong... with an Inquiry into its History in European Literature. Chicago 1944; A. Huon, Le Roy Sainct Panigon dans l'imagerie populaire du $X V f$ siècle, in: François Rablais, Ouvrage publié pour le quatrième centenaire de sa mort 1553-1953, Genève - Lille 1953, pp. 210-225.

${ }^{7}$ Cucania in latino; Coquaigne, Cocagne in francese; Cucaña in spagnolo; Cokaygne in inglese, ecc. In Germania si disse Schlauraffenland, Schlaraffenland; nelle Fiandre Luilekkerland. Cfr. A. Graf, op. cit., p. 232 , note.

${ }^{8}$ Ibidem, p. 232. 
del paese di Cuccagna. Una rappresentazione di Cuccagna molto interessante è contenuta nel Capitolo di Cuccagna del 1581 ed nel Trionfo dei poltroni, sempre del Cinquecento. Le meraviglie del paese di Cuccagna ci sono dipinte in ventitré strofe in una stampa del 1588 conservata al British Museum intitolata $1 /$ piacevole viaggio di Cuccagna. Di nuovo ritrovato, et stampato a commodità di tutti $i$ bon compagni, che desiderano andare in quel paese. $\mathrm{E}$ infine dal Seicento proviene $I l$ Trionfo di Cuccagna, opera di Martin, cieco di Lucca'.

Il paese di Cuccagna ha i suoi prototipi e le sue analogie, fra cui la visione del paradiso terrestre che sta in stretta relazione con quella del paese di Cuccagna. Entrambe le immaginazioni nascono dallo stesso principio, dallo stesso desiderio, dallo stesso sogno di felicità, i quali, se variano quanto a certe apparenze e a certi caratteri, nella sostanza rimangono pur sempre invariati ${ }^{10}$. Il paradiso terrestre e la Cuccagna sono due termini diversi, ma non contraddittori, a cui riesce lo stesso pensiero, secondo l'affetto che lo muove, e in conformità della mente entro la quale si muove, notò Novati ${ }^{11}$. Anche i paradisi delle religioni inferiori sono veri paesi di Cuccagna. Così i greci ebbero l'età dell'oro e dei Campi Elisi, ma anche quella di una terra felice che mostra con la Cuccagna grandissima somiglianza, essendo tuttavia una parodia dei Campi Elisi ${ }^{12}$. Il mito dei Campi Elisi pare che sia una fonte diretta da cui traggono ispirazione i paesi di Cuccagna ${ }^{13}$. Luciano ad esempio, descrive nella Vera Istoria la città dei beati, la quale è „tutta d'oro con le porte di cinnamomo, il suolo d'avorio, i templi di berillo, gli altari d'ametista. Cinge la città un fiume d'ottimo unguento, largo cento cubiti, profondo cinquanta. Le terme sono grandi palazzi di cristallo, dove, il luogo di acqua si adopera rugiada riscaldata. Quivi non è mai notte, né dì, ma un lume mitissimo, quale si ha il mattino, prima del levare del sole; né altra stagione vi si conosce che la primavera, né altro vento che il zeffiro. Abbondano in quella terra piante bellissime d'ogni qualità e che mai non cessano di far frutto. Le viti si coprono di grappoli dodici volte l'anno; le spighe del grano, in luogo di chicchi, recano pani. Intorno alla città sono trecentosessantacinque fontane d'acqua, altrettante di miele, cinquecento di vari unguenti, ma più piccole, sette fiumi di latte, otto di vino. L'Elisio è un campo bellissimo, cinto da una selva di grandi alberi vitrei, che recano per frutti coppe di varie forme e grandezze. Chi vuol bere non ha che a spiccarne una, la quale tosto si colma di vino. Dense nubi assorbono dalle fontane e dal fiume gli unguenti, e premute da lievi aure, li versano in rugiada"14. Il mito fu certamente note anche ai Latini, sebbene nella loro letteratura non lo si trovi ricordato in modo esplicito. Nei romanzi persiani si trovano spesso memorie di un paese Sciadukaim, che non è affatto

${ }^{9}$ Cfr. G. Cocchiara, op. cit., pp. 160-171; P. Camporesi, op. cit., pp. 228-232.

${ }^{10}$ A. Graf, op. cit, p. 229.

${ }^{11} \mathrm{~F}$. Novati, /l paese che non si trova, La domenica letteraria, anno IV, n. 11, 15 marzo 1885.

${ }^{12}$ A. Graf, op. cit., p. 230.

${ }^{13}$ G. Cocchiara, op. cit., p. 178.

${ }^{14}$ Vera Istoria, I. II, capp. 11-14; si cita da A. Graf, op. cit., pp. 230-231. 
diverso dal paese di Cuccagna ${ }^{15}$. Le meraviglie del paese di Cuccagna sono state anche messe a confronto con il paradiso cristiano e la differenza che ne risulta è tale che l'immagine delle delizie del paradiso suscita nelle anime devote da un desiderio di felicità e di innocenza mentre l'immagine delle delizie del paese di Cuccagna viene animata, in tutti gli affamati, da un desiderio più particolare di liberarsi dagli stenti, di appagare gli appetiti più animaleschi ${ }^{16}$. Infine, è molto probabile, sottolinea Graf, che i vari paradisi artificiali, quali ad esempio i giardini sospesi di Babilonia, volessero essere una riproduzione del paradiso assiro e quindi una versione del paese di Cuccagna ${ }^{17}$.

Con l'osservazione sui paradisi artificiali in quanto realizzazioni del paese di Cuccagna siamo giunti al punto in cui giova accennare alla pubblicità. Come hanno notato Wiesław Szpilka e Dariusz Czaja ${ }^{18}$ le immagini pubblicitarie come nel caso del catalogo pubblicitario dell'azienda Schuster, analizzato da Szpilka o quello degli spot del sapone Palmolive, della Renault Clio o del cioccolatino Bounty presi in esame da Czaja, riproducono visioni del paradiso. Le immagini pubblicitarie sono una realizzazione contemporanea dell'immagine del paradiso, sono una rivelazione dell'archetipo del paradiso impresso nella immaginazione dell'uomo.

In seguito vorremmo appunto dimostrare che le immagini della pubblicità televisiva italiana degli anni novanta possono essere riconosciute come una visione moderna di Cuccagna, sorta di terra sognata di felicità. Il mito del paese di Cuccagna, così vivo nel Medioevo e nel Rinascimento in Italia, alimenta fino ad oggi l'immaginazione delle persone che sognano una realtà migliore e, che oltretutto, la vedono promessa nella pubblicità; ma così come Cuccagna anche il mondo offerto dalla pubblicità è un paese che non si trova.

\section{LA PUBBLICITẢ TELEVISIVA ITALIANA COME REALIZZAZIONE DEL MITO DEL PAESE DI CUCCAGNA}

La popolarità di questo mito tra gli italiani si spiegherebbe probabilmente grazie all'osservazione di Novati, ripetuta ulteriormente da Cocchiara, il quale sottolinea l'importanza che per il popolo italiano assume il desiderio intenso di una felicità materiale, che si esplica nella soddisfazione piena, illuminata da ogni sensuale appetito ${ }^{19}$.

${ }^{15}$ A. Graf, op. cit., pp. 231-232.

${ }^{16}$ Ibidem, p. 234.

${ }^{17}$ lbidem, p. 237.

${ }^{18} \mathrm{Cfr}$. W. Szpilka, Raj nieutracony, in: Mitologie popularne. Szkice z antropologii współczesnej, a cura di D. Czaja, Kraków 1994, pp. 129-141; D. Czaja, Reklamowy smak raju, in: Mitologie, op. cit., pp. 143-158.

${ }^{19}$ G. Cocchiara, op. cit., p. 159. 
Troviamo questa considerazione molto arguta, giusta e sempre attuale come si rivela anche nella pubblicità analizzata nelle cui parole ed immagini vediamo le principali caratteristiche del paese di Cuccagna. Prima comunque di addentrarci nella presentazione degli esempi, vorremmo ricordare ciò che Marco Giusti ha detto a proposito di Carosello. Esso „vendeva la gioia di consumo, l'idea che la vita potrebbe essere non una valle di lacrime, ma un albero della Cuccagna, e faceva conoscere $i$ prodotti in un'aura di festosità, di allegria, di scherzo, garantiti dalla celebrità del momento" ${ }^{20}$. A nostro giudizio la pubblicità televisiva italiana degli anni novanta è fondata sullo stesso principio di mostrare il mondo come una favola, un paese di felicità, un paradiso, insomma, un vero e proprio paese di Cuccagna.

Dopo la lettura delle descrizioni del paese di Cuccagna fornite dai testi sopra nominati abbiamo individuato sei caratteristiche cardinali di questa terra mitica. La prima ed la più spettacolare è che vi sia un'abbondanza di cibo e bevande sterminata, facilmente accessibile a tutti. Ciò è legato ad un'altra particolarità apprezzabile di Cuccagna: i prodotti vi si vendono a buon mercato o addirittura si regalano. Poi, questo è un paese di riposo, non vi si lavora mai, anzi, la regola che vi regna è quella che chi più dorme, più guadagna. Inoltre, a Cuccagna è sempre festa ed è sempre tempo di gioia. Infatti, belle donne procurano a chi passa per il paese, tutto il piacere sensuale che desidera. Ed in ultimo luogo, nel paese di Cuccagna le differenze tra le persone non valgono più: sono tutte uguali.

Ora cercheremo di illustrare i suddetti caratteri del paese di Cuccagna sia in relazione ad alcuni brani dei testi letterari italiani già menzionati che alle pubblicità provenienti dal campione preso in esame. Così miriamo a dimostrare le analogie tra la tradizionale immaginazione del paese di Cuccagna e le immagini pubblicitarie.

Come si è detto prima, ciò che balza subito agli occhi al primo sguardo è l'abbondanza di cibi e bevande. Notiamo questa caratteristica di Cuccagna a partire da Boccaccio nella cui Bengodi ,si legano le vigne con le salsicce (...), ed eravi una montagna tutta di formaggio parmigiano grattugiato, sopra la quale stavan genti che niuna altra cosa facevan che far maccheroni e raviuoli e cuocerli in brodo di capponi, e poi li gettavan quindi giù, e chi più ne pigliava più se ne aveva; e ivi presso correva un fiumicel di vernaccia della migliore che mai si bevve, senza avervi entro goccio d'acqua"21. Un simile aspetto ha Cuccagna descritta da Faustino da Terdoccio:

(..) e quando uno è affamato

li piove manna in bocca

e spesse volte i fiocca

i sacchi di confetti

${ }^{20}$ Carosello. Non è vero che tutto fa brodo 1957-1977, Catalogo di in occasione del $70^{\circ}$ anniversario di Sipra, a cura di Marco Giusti, Silvana Editoriale, 1996, p. 110.

${ }^{21}$ Boccaccio, Decamerone, giorn. VIII, nov. 3. 
e son coperti i tetti

de zalde inzuccherate ${ }^{22}$.

Lo afferma anche il poeta ebreo cinquecentesco di cui si è parlato: „Questa è la terra di latte e di miele....23 Invece nel Capitolo di Cuccagna del 1581 leggiamo:

O quanta bella grascia, e buoni vini

starne, fagiani, e carne di porcelli

gredi, vernaccia, malvasia e latini ${ }^{24}$ !

L'autore de $I l$ piacevole viaggio di Cuccagna incontra in questo paese, tra le altre attrazioni, la montagna di Bacco dove:

(...) i capon, le pernici ed i fagiani

arrosto, allesso, intingoli e guazzetti

son poco in pregio, ché di più soprani

cibi ci son, con vini e di più perfetti.

Or qui i denti menar si può e le mani

senza riguardo, o aver tanti rispetti,

ché non si guarda più crudo che cotto,

e più in stima è ché più imbriaco e giotto ${ }^{25}$.

Per finire ricordiamo un brano sullo stesso argomento tratto da Il Trionfo di Cuccagna, opera di Martin, cieco di Lucca:

Son coperti i tetti di cialde inzuccherate

Lasagn informaggiate si gettan per la via

Ci è tanta malvagia, ch'ognun s'è imbriacato

Oh! Quanta pinocchiata! Ne ingrassano i porcelli

Ci è tanti biscotti, che ne empiono le fosse ${ }^{26 !}$.

L'abbondanza di cibi e bevande e la facilità con cui sono accessibili si manifesta anche nella pubblicità televisiva italiana bensì in maniere diverse adeguate all'immaginario moderno e alle tecniche di raffigurazione possibili nella pubblicità. Innanzitutto, vale la pena notare che gli spot dei prodotti alimentari occupano la maggior parte del tempo pubblicitario, come rivela il campione da noi analizzato con cinquantacinque filmati su cento che riguardano cibi e bevande senza contare l'acqua minerale e con trentaquattro prodotti alimentari su cinquantotto di tutti quelli inclusi nello schedario. Esso, infatti, si presenta come se fosse il menu di una grande tavola imbandita, una di quelle che s'incontrano nel paese di Cuccagna, nella quale si trovano diversi tipi di pasta, quali Penne tricolore Findus, Pasta cuore, Anolini Buitoni, Spianarelle Barilla e Spaghetti Barilla; i diversi tipi di con-

\footnotetext{
${ }^{22}$ Si cita da G. Cocchiara, op. cit., p. 163.

${ }^{23}$ Si cita da ibidem, p. 162.

${ }^{24}$ Ibidem, p. 167.

${ }^{25}$ Si cita da P. Camporesi, op. cit, p. 231.

${ }^{26}$ Si cita da G. Cocchiara, op. cit, p. 170.
} 
dimenti: Sugo al basilico Barilla, Condiriso Sasso e Dado Star; poi, le zuppe come Zuppa del casale e Zuppa del casale ortolana Findus; formaggi: tre tipi di Philadelphia, Parmigiano Reggiano e Certosa Galbani; il Prosciutto di Parma; prodotti del tipo pane quali Fette biscottate Mulino Bianco e Cracker Mulino Bianco. In seguito vi è anche frutta e verdura: Verdure Orogel e Melinda; e come dessert i dolci: Flauti Mulino Bianco, Pan di stelle Mulino Bianco, Morbidelli Sperlari, Gelatine Sperlari, Torrone Sperlari, Gran Nocciolato Maina, Panettone Bauli e Emozioni Perugina. Da bere invece si serve latte: Granarolo latte; caffè: tre tipi di caffè Lavazza ed il caffè Segafredo; e alcolici quali Etichetta Nera, Martini e Campari Soda. Lo spettatore, alla stessa stregua del viaggiatore nel paese di Cuccagna, viene invitato a servirsi di queste bontà. La pubblicità italiana appare qui come un enorme banchetto virtuale. Il cibo ci sta sempre, e poi sempre abbondante e ideale: il pane è sempre fresco, il vino di qualità, la carne sanissima, le verdure genuine, i dolci unici ecc.

A parte l'aspetto metaforico di una tavola imbandita, l'idea dell'abbondanza si spiega nella pubblicità televisiva italiana tramite alcune immagini o parole. Quasi ogni spot di qualsiasi prodotto alimentare contiene inquadrature con ingrandimenti della vivanda o moltiplicazioni di confezioni di essa. In un filmato Lavazza, addirittura, i pacchetti di caffè, come la manna, cadono in grandi quantità dal cielo. Uno degli spot Mulino Bianco inizia con una ripresa di un cesto da spesa pieno che occupa tutta l'inquadratura e ci fa pensare al mitologico como dell'abbondanza. In uno spot Parmigiano Reggiano abbiamo alcune riprese di magazzini riempiti di fomaggio, in un altro invece arriva un furgone carico di grandi e appetitosi pezzi di parmigiano. Lo slogan dello spot Gran Nocciolato Maina ci insegna verbalmente con quanta quantità di delizie abbjamo a che fare: „Un mondo di bontà". Nella pubblicità Gelatine Sperlari anche le mucche mangiano le caramelle (ricordiamo che in Cuccagna „danno i confetti insino a' cani" ${ }^{\text {,27) }}$ ed il panettone Bauli basta per tutti i numerosi protagonisti del filmato. Lo spot Orogel ci fa passare davanti agli occhi molte delle buone verdure di cui si compongono i suoi prodotti. La réclame di Pasta cuore è ambientata tra le numerose, coloratissime bancarelle di un mercato abbondantemente caricate. Barilla invece propone delle immagini del tavolo di un banchetto di nozze riccamente imbandito oppure un'invitante tavolata dal sindaco.

Come si è già detto il cibo nel paese di Cuccagna non era solo presente in abbondanza e in ogni luogo ma era quasi gratuito. Vediamo alcuni brani che ne parlano e che, d'altronde, sembrano, di per sé, una specie di pubblicità. Boccaccio dice ad esempio: „(...) Avevavisi un'oca a denaio e un papero giunta” ${ }^{28}$. Faustino da Terdocio sottolinea invece:

e cento marzapani

se dan per un quatrino

e con un sol terlino

${ }^{27}$ Il piacevole viaggio di Cuccagna, in: op. cit., p. 232.

${ }^{28}$ Boccaccio, Decamerone, giorn. VIII, nov. 3 . 
se compra un vitel cotto

con certo buon biscotto

ch'è de pan mellato... ${ }^{29}$

Se diamo retta all'autore de Il piacevole viaggio di Cuccagna possiamo viaggiarvi „Senza che pur spendiate un quatrino" perché vi è a un'osteria che:

(...) osteria dei sempi vien chiamata

non molto da Mattelica discosto,

dove la mensa è sempre apparecchiata

con delicati cibi arrosto e allesso;

mangiato ch hai con tutta la brigata

sei franco, né si fa conto con l'osto,

perché è provigionato dal paese

che faccia a viandanti buone spese.

E inoltre:

(...) chi non ha dinar, tutti securi

trovarà i passi, gli alberghi e i spedali,

e arriverà in Cuccagna alla distesa

senza un soldo pegar di dazio o spesa $a^{30}$.

Martin, cieco di Lucca in Il Trionfo della Cuccagna ci assicura che vi sono "delle salsicce grasse a dui quattrini il cento" ${ }^{\text {"31 }}$.

È facile ricordarsi che incontriamo uguali promesse di acquisto dei vari prodotti a buon mercato nel mondo creato dalla pubblicità, e in questo caso, non solo da quella italiana. Dei prezzi spesso non si parla neanche come se tutto fosse gratuito, o, eventualmente, si garantisce che con "sole....lire" oppure „spendendo pochissimo" possiamo regalarci tutto ciò che desideriamo.

Poi, una caratteristica molto aprezzata del paese di Cuccagna è quella che non vi si lavora mai. È la terra di un incessabile riposo, anzi, chi più dorme più guadagna. Il Capitolo di Cuccagna vanta ciò in questi termini:

Sono stato nel Paese di Cuccagna

quante belle usanze son fra loro!

Quello che più ci dorme ci guadagna.

(...)

Là non ci parlar mai di lavorare che subito ti mettono in prigione e un anno intero ti ci fan stare . $^{32}$.

\footnotetext{
${ }^{29}$ Op. cit., cfr. nota 9 .

${ }^{30}$ Op. cit., cfr. nota 25, p. 229.

${ }^{31}$ Op. cit., cfr. nota 9.

${ }^{32} \mathrm{Jbidcm}$.
} 
Il piacevole viaggio di Cuccagna inizia invece con questo invito:

Venite spensierati e compagnoni,

voi ch'avete si in odio il lavorare,

amici delli grassi e buon bocconi.

nemici del disagio e del stentare.

Omini di gran cor, non già poltroni.

come gli avari vi voglio(n) chiamare,

venite tutti, che andiamo in Cuccagna

dove chi più dorme più guadagna ${ }^{33}$.

E altrove:

Promettendo a costor sua fede ancora

che non ti venirà più fantasia

di lavorar né di stentar un'ora

potendo star su la poltroneria,

e in vita tua mandar a la mal'ora

gli impacci, i stenti e la maninconia,

godendo fin che pôi allegramente

col corpo pieno e ben bagnato il dente ${ }^{34}$.

Nel mondo ideato dalla pubblicità televisiva italiana non si lavora neppure. Non vi è una sola immagine di fatica o di stento. Tutte le persone sono rilassate, contente e felici mentre si dedicano agli svaghi a tavola, a casa o al ristorante, ai giochi, ai divertimenti, ai piaceri di ogni genere. Perfino i protagonisti che in teoria dovrebbero svolgere alcune manzioni, quali il Maresciallo o l'Ispettore di Pagine Gialle o il medico di Wind come pure gli operatori di Telecom Italia, si divertono, e con un sorriso ci fanno intendere che nel mondo della pubblicità televisiva italiana, la loro occupazione anziché un lavoro è un vero piacere. Inoitre, così come nel paese di Cuccagna l'uva produce da sola il vino ${ }^{35}$ nella pubblicità televisiva italiana il grano produce da solo le fette biscottate senza che l'uomo intervenga (Fette biscottate Mulino Bianco), le verdure si seminano e crescono da sole (Orogel) oppure un dado di brodo si "spoglia" da solo, i pomodori si tagliano e si trasformano in sugo (Dado Star), mentre l'uomo si riposa o mangia. Nello stesso modo, con l'aiuto della magia, in un niente, si fanno „dodici prelibate ricette di pollo, preparate in porzioni da uno. Tanti piaceri diversi per uno", per tutti i cavalieri della tavola rotonda, senza lavorare (Garbini ). In un'ottica più realistica, la pubblicità televisiva italiana propone prodotti che ci liberano dalla fatica, come ad esempio fanno i sughi pronti (Sugo al basilico Barilla).

Nel paese di Cuccagna non vi è posto per il lavoro e la fatica, ma vi è tutto il tempo per i festeggiamenti e la gioia.

\footnotetext{
${ }^{33}$ Ibidem, p. 228.

${ }^{34}$ Op. cit., p. 231.

${ }^{35}$ Capitolo di Cuccagna, in: op. cit, p. 167.
} 


\section{Sempre v'è tempo gratioso e sano}

non ci vedi altro se non festeggiare

non si pagan le biade, né anco il grano ${ }^{36}$.

Scrive l'autore di Capitolo di Cuccagna, mentre Graf ricorda: „vi si celebrano quattro pasque, e quadruplicate sono l'altre feste principali, mentre la quaresima viene solo una volta ogni vent'anni"37.

I festeggiamenti di vario tipo sono uno degli aspetti fondamentali della creazione della realtà nella pubblicità televisiva italiana. Inoltre, la festa viene sfruttata dalla pubblicità in molti modi: in quanto riferimento ad una festa particolare, in quanto rappresentazione dell'insieme delle esperienze e delle emozioni connesse al vivere la festa ed infine in quanto festa stessa. A proposito del coinvolgimento della festa nel linguaggio pubblicitario Claudio Bernardi scrive in questi termini: „Nell'immagine pubblicitaria la festa costituisce il mito di fondo, il grande racconto che contiene e caratterizza i mille episodi di consumo e che si presenta in molti modi, come magico Natale, festa con gli amici, domenica in famiglia, Paese di Cuccagna, Isole Vergini, sagra campestre, vestito e festa, corpo glorioso, terra promessa" ${ }^{38}$. Effettivamente alla stessa stregua di Cuccagna, tutte le feste possibili sono rilevate, celebrate, in qualche modo quadruplicate o moltiplicate. La quaresima non giunge neanche una volta ogni vent'anni, essa viene scordata completamente.

Le immagini pubblicitarie in questione attingono spesso ed in grandi quantita alle feste di diversi tipi: arcaiche, ebree, cristiane, borghesi. Dato però che l'argomento oltrepassa di gran lunga l'idea di questo articolo, ci limitiamo solo a questo breve accenno.

Tuttavia, l'immagine della festa nella pubblicità televisiva italiana non si esaurisce nell'elenco dei tipi individuati ma comprende ben altri aspetti.

La festa significa la gioia, il tripudio, il riso, lo sfogarsi, l'effervescenza, l'eccesso ecc. R. Caillois dice che la festa ,si l'on ne considère que ses aspects extérieures, présente des caractères identiques à n'importe quel niveau de civilisation. Elle implique un grand concours de peuple agité et bruyant. Ces rassemblements massifs favorisent éminemment la naissance et la contagion d'une exaltation qui se dépense en cris et en gestes, qui incite à s'abbandonner sans contrôle aux impulsions les plus irréfrechies. (...) De jadis ou d'aujourd'hui, la fête se définit toujours par la danse, le chant l'ingestion de nourriture, la beuverie" ${ }^{39}$. La pubblicità televisiva italiana è piena di tali manifestazioni festive ed il campione analizzato lo dimostra. Sono poche le pubblicità in cui non si rida o balli o manifesti la propria

${ }^{36}$ Ibidem.

${ }^{37}$ A. Graf, op. cit., p. 233.

${ }^{3 *}$ Voce Festa in: Dizionario della pubblicita. Storia, tecniche, personaggi, a cura di A. Abruzzcse, F. Colombo, Zanichelli 1994, p. 187.

${ }^{39}$ R. Caillojs, $L$ homme et le sacré, Gallimard, Paris 1950, pp. 123-124. 
contentezza, o non si beva o mangi. La pubblicità televisiva italiana dà l'impressione di essere in sé una grande festa.

Vi sono più motivi per cui ci pare opportuno avanzare tale tesi.

Bronislaw Geremek ha scritto che le feste nel Medioevo costituivano, per un attimo, per un momento transitorio, una realizzazione dei sogni più profondi degli uomini di allora: i sogni di una vita libera dal ritmo implacabile del lavoro, libera dalle preoccupazioni quotidiane, una vita non sottomessa ai detti della dottrina morale, una vita piena di allegria, di abbondanza di cibi e bevande, senza divisioni sociali ${ }^{40}$. L'analogia si impone da sola: anche la pubblicità mira a realizzare i nostri sogni i quali nella dimensione concreta si differenziano da quelli medievali ma in genere si tratta sempre dello stesso sogno di una vita senza obblighi, senza preoccupazioni, allegra e rilassante. E per quanto la festa medievale fosse un surrogato di tale esistenza la pubblicità contemporanea ne crea l'illusione. Il resto lo compiamo noi spettatori nel processo dell'identificazione con le immagini proposte ${ }^{41}$.

Poi, a proposito della festa in una società tradizionale $P$. Burke scrive in questi termini: „la festa si opponeva al quotidiano, tempo di spreco proprio perché il quotidiano era tempo di scrupoloso risparmio"42. In questo senso pure la pubblicità s'avvicina alla festa tradizionale popolare. La pubblicità, come si sa, di per sé è un invito ad acquistare e quindi a spendere, a non risparmiare, allo spreco e infine al godimento del bene materiale. Non di rado cediamo a questa realtà di festa commerciale.

In ultimo luogo vorremmo accostarci all'aspetto dei piaceri sensuali di Cuccagna. Lì abitano bellissime donne che riscaldano i cuori dei viaggiatori e procurano loro la gioia con la loro presenza:

Al fine poi della selva è una pianura

lunga quanto può uscir un stral di cocca;

quivi è una villa cosi amena e pura,

con bei ruscelli e fontanine in chiocca

che invita a riposar ciascun ch'arriva

con qualche bella e graziosa diva.

Abitan donne sole in questo loco,

che ti vengon a far grata accolienza

scaldano il cor tuo d'amoroso foco

coi gesti, col parlar, con la presenza.

Or poi che qui sei rificiato un poco

e mangiato hai di buon a sufficienza,

doi d'esse a tua elezion ti poi capare

e seco starti in gioia a riposare ${ }^{43}$.

${ }^{40}$ B. Gercmek, Życie codzienne w Paryżu Franciszka Villona. PIW, Warszawa 1972, p. 215.

${ }^{41}$ Cfr. U. Eco, La struttura assente, Tascabili Bompiani 1985, p. 169.

${ }^{42}$ P. Burke, op. cit., p. 174.

${ }^{43}$ Il piacevole viaggio di Cuccagna, in: op. cit., p. 231. 
Sicuramente belle e graziose dive nonché allusioni ai piaceri sensuali o, perfino, sessuali non mancano negli spot pubblicitari italiani di cui stiamo parlando. A rallegrare l'occhio dello spettatore e riscaldargli il cuore vi sono schiere di perfette modelle le quali a volte imitano famosi star quali Marilyn Monroe e Rita Hayworth nei filmati Paradiso altre volte sono invece vere dive del cinema italiano, come Matilde Koll dello spot Pagine gialle. Comunque il motivo del possibile piacere sensuale e sessuale si realizza nella pubblicità televisiva italiana in due temi principali: primo, mostrando la donna come oggetto sessuale o in ogni caso visibilmente rappresentata nei suoi lati più attraenti; secondo, mostrando scene di seduzione maschile, cogliendo quel particolare atteggiamento di seduzione dell'uomo nei confronti della donna. Entrambi i temi si riassumono in un ruolo attribuito alla donna, vale a dire, quello della possibile amante.

Vedere una bella donna è di per sé un piacere sensuale di cui testimoniano secoli di evoluzione dell'arte. La donna, così come viene presentata dalla pubblicità televisiva italiana, cioè con i sui attributi sessuali (grande seno, fianchi formosi e cosce lunghe) ben in evidenza, diventa una promessa di piacere ulteriore, quello sessuale. Tali „dive graziose" le incontriamo anche all'infemo (Segafredo) dove le diavolette, belle e ben fatte, attirano con i loro vestiti aderenti e trasparenti. Molto "sexy" è altresì la Befana della pubblicità italiana (Wind), la quale, tradizionalmente vecchia e brutta, si rivela qui come una bella e formosa mora in pantaloni e maglietta neri aderenti. Un simile oggetto di desiderio lo incontriamo dal medico di un altro spot, Wind, e si accompagna subito, appositamente, all'esclamazione: „Bella tariffa!". Le due modelle della pubblicità Omnitel si inseriscono con molta facilità in questo tipo di donna "da piacere".

D'altra parte nella pubblicità italiana vi sono non pochi accenni alla seduzione maschile. Essa si verifica anche in paradiso, dove l'Uomo „ci prova" dapprima con Rita Hayworth - Paradiso -- poi con Marilyn Monroe ed infine anche con un angelo di sesso femminile. Nello spot Martini il giovane riesce a sedurre la giovane amante del vecchio e mentre l'uomo Buitoni (Buitoni) usa la seduzione come modo per carpire la ricetta degli agnolini. Un'allusione sessuale appare anche in alcuni contesti che sembrerebbero del tutto innocenti. Il Raimondo del filmato Melinda, mentre strappa una piccola etichetta da una mela, è accompagnato dal commento fuori campo: „sta spogliando Melinda”. Melinda, potendo essere un nome femminile contribuisce a creare un doppio senso, rafforzato dallo slogan pronunciato con una voce molto sensuale: „Tutti pazzi per Melinda”.

Come vediamo il piacere sensuale procurato dalle donne nella pubblicità televisiva italiana degli ultimi dieci anni e quello sessuale promesso dalle sue immagini costituiscono un altro legame tra le tradizionali immaginazioni del paese di Cuccagna ed il mondo ideato da questa pubblicità.

Speriamo di essere riusciti a dimostrare efficacemente fino a che punto questi due paesi immaginari, uniti dallo stesso desiderio di felicità nei termini di soddisfazione materiale e sensuale, pur essendo lontani nel tempo, siano 
vicini l'uno all'altro e provino l'immortalità ovvero l'estrema longevità di alcune tendenze dell'immaginario comune, di alcuni archetipi pure, e di mentalità entro una nazione.

D'altra parte, il sogno di felicità che una volta si realizzava nel paese di Cuccagna non è diverso, in molte particolarità, da quello offerto dalla pubblicità italiana.

\section{BIBLIOGRAFIA}

Burke P. (1980), Cultura popolare nell'Europa moderna, Mondadori, Milano.

Camporesi P. (1978), Il paese della fame, 11 Mulino, Bologna.

Cocchiara G. (1981), Il mondo alla rovescia. Boringhicri, Torino.

Graf A. (1892), Il paese di Cuccagna e i paradisi artificiali, in: Miti, leggende e superstizioni del Medio Evo, Loescher, Torino.

Novati F. (1885), Il paese che non si trova, La domenica letteraria, anno IV, n. 11, 15 marzo. 